Mathematical Research Letters 10, 423-434 (2003)

\title{
INTEGRALLY CLOSED IDEALS IN TWO-DIMENSIONAL REGULAR LOCAL RINGS ARE MULTIPLIER IDEALS
}

\author{
Joseph Lipman and KeI-ICHI Watanabe
}

\begin{abstract}
Multiplier ideals in commutative rings are certain integrally closed ideals with properties that lend themselves to highly interesting applications. How special are they among integrally closed ideals in general? We show that in a two-dimensional regular local ring with algebraically closed residue field there is in fact no difference between "multiplier" and "integrally closed" (or "complete.") But among multiplier ideals arising from an integer multiplying constant (also known as adjoint ideals), and primary for the maximal ideal, the only simple complete ideals are those of order one.
\end{abstract}

\section{Introduction}

There has arisen in recent years a substantial body of work on multiplier ideals in commutative rings (see [La]). Multiplier ideals are integrally closed ideals with properties that lend themselves to highly interesting applications. One is tempted then to ask just how special multiplier ideals are among integrally closed ideals in general.

In this note we show that in a two-dimensional regular local ring $R$ with maximal ideal $\mathfrak{m}$ such that the residue field $R / \mathfrak{m}$ is algebraically closed ${ }^{1}$ there is actually no difference between multiplier ideals and integrally closed ideals. In fact it turns out more convenient to do this for fractionary $R$-ideals, i.e., nonzero finitely-generated $R$-submodules of the fraction field $L$ of $R$.

\section{Main Result}

Every integrally closed fractionary R-ideal is a multiplier ideal.

After this paper was first submitted, we learned that independently of us C. Favre and M. Jonsson had found a related proof [FJ, §6]. Their argument is

\footnotetext{
Received December 1, 2002.

2000 Mathematics Subject Classification. 13B22, $13 \mathrm{H} 05$.

First author partially supported by the National Security Agency. Second author partially supported by Grants-in-Aid in Scientific Researches, 13440015, 13874006; and his stay at MSRI was supported by the Bunri Fund, Nihon University. Both authors are grateful to MSRI for providing the environment without which this work would not have begun. Research at MSRI is supported in part by NSF grant DMS-9810361.

${ }^{1}$ It most likely suffices that $R / \mathfrak{m}$ be infinite, but we want to avoid additional technicalities.
} 
given in the context of a novel treatment of valuations of $R$. Though we thought initially that our proof applied only to m-primary ideals, Favre and Jonnson had no such restriction. This prompted us to reexamine our proof, which we then found could be made to apply to the general case as well.

Throughout, $(R, \mathfrak{m})$ and its fraction field $L$ will be as above. "Ideal" will mean "fractionary $R$-ideal." An ideal is integral if it is contained in $R$; and of finite colength if it is either (integral and) m-primary or the unit ideal. For brevity we use the classical term "complete" instead of "integrally closed in $L$." Any complete ideal $I$ is uniquely the product of a principal ideal and a finitecolength complete ideal: $I=\left(I^{-1}\right)^{-1}\left(I I^{-1}\right)$ where the inverse of an ideal $J$ is $J^{-1}:=\{x \in L \mid x J \subset R\}$.

\section{Geometric formulation of the problem}

The goal of this section is to develop the geometric criterion Corollary 1.4.2 for an ideal to be a multiplier ideal, while laying the groundwork for the proof in the next section that every complete ideal satisfies that criterion.

We begin by recalling some preliminary definitions and known results. (For some historical pointers to the development of the theory of complete ideals see the second paragraph on the first page of [L3].)

For any complete ideal $I$ there exists a $\log$ resolution, that is, a proper birational map $f: X \rightarrow \operatorname{Spec}(R)$ where $X$ is a regular scheme such that for any closed point $x \in X$ there exist $t_{1}, t_{2}$ generating the maximal ideal of $S:=\mathcal{O}_{X, x}$ such that $I S=t_{1}^{a_{1}} t_{2}^{a_{2}} S$ for some integers $a_{1}, a_{2}$. (In other words, $I \mathcal{O}_{X}$ is invertible, with normal-crossing support.) To see quickly that there is an $f$, composed of maps obtained by blowing up closed points, and such that the $\mathcal{O}_{X}$-ideal $I \mathcal{O}_{X}$ is at least invertible - assuming, as one clearly may, that $I$ is $\mathfrak{m}$-primary - one can use the Hoskin-Deligne formula [L2, p. 222, Thm. 3.1], which shows that the length of the "transform" of $I$ can be successively lowered by suitable closed-point blowups, until it vanishes, at which point $I$ generates an invertible ideal sheaf. Then well-known facts about embedded resolution of curves in two-dimensional regular schemes ensure that with further closed-point blowups one can reach the desired normal-crossing situation.

The group $\operatorname{Div}(X)$ of $X$-divisors is, by definition, the free abelian group on the set of reduced irreducible one-dimensional subschemes of $X$. These subschemes, also called prime divisors, are of two kinds, affine resp. exceptional according as $f$ maps their generic point to a non-closed resp. closed point of $\operatorname{Spec}(R)$. A divisor $D$ can be represented as a formal sum with integer coefficients, $D=\sum_{E} d_{E} E$ where $E$ runs through all prime divisors and only finitely many of the integers $d_{E}$ are nonzero; an $E$ for which $d_{E} \neq 0$ will be called an irreducible component of $D$. One has then the decomposition $D=D_{\text {aff }}+D_{\text {exc }}$ where $D_{\text {aff }}$ (resp. $D_{\text {exc }}$ ), the affine (resp. exceptional) part of $D$ is obtained by replacing all the $d_{E}$ where $E$ is exceptional (resp. affine) by 0 . We say $D$ is affine (resp. exceptional) if $D=D_{\text {aff }}$ (resp. $D=D_{\text {exc }}$ ). Exceptional divisors will also be called $f$-divisors. They make up a subgroup $\operatorname{Div}_{\mathrm{e}}(X) \subset \operatorname{Div}(X)$. 
Each prime divisor $E$ gives rise to the discrete valuation $v_{E}$ whose valuation ring is the local ring on $X$ of the generic point of $E$ (which may be assumed to be a subring of $L$ ). The divisor $(t)$ of a nonzero $t \in L$ is defined to be $(t):=\sum_{E} v_{E}(t) E$. One has $t \in R \Leftrightarrow v_{E}(t) \geq 0$ for all affine $E \Leftrightarrow(t)_{\text {aff }} \geq 0$.

For $D=\sum_{E} d_{E} E$, the sheaf $\mathcal{O}_{X}(D)$ is the invertible $\mathcal{O}_{X}$-module sending any open $U \subset X$ to

$$
\Gamma\left(U, \mathcal{O}_{X}(D)\right):=\left\{t \in L \mid v_{E}(t) \geq-d_{E} \text { for all } E \text { meeting } U\right\}
$$

In particular, $\Gamma\left(X, \mathcal{O}_{X}(-D)\right):=\{t \in L \mid(t) \geq D\}$. This is a complete ideal, integral iff $d_{E} \geq 0$ for all affine $E$, and of finite colength iff $D$ is exceptional. Every invertible $\mathcal{O}_{X}$-submodule of the constant sheaf $L$ is $\mathcal{O}_{X}(D)$ for a unique $D$.

The abelian group $\operatorname{Div}(X)$ is ordered, the positive (or effective) divisors being those $D$ such that $d_{E} \geq 0$ for all $E$, or equivalently, $\mathcal{O}_{X}(-D) \subset \mathcal{O}_{X}$. An effective $D$ can be regarded as a one-dimensional subscheme of $X$, with structure sheaf $\mathcal{O}_{D}$ fitting in a natural exact sequence

$$
0 \rightarrow \mathcal{O}_{X}(-D) \rightarrow \mathcal{O}_{X} \rightarrow \mathcal{O}_{D} \rightarrow 0
$$

If, moreover, $D$ is exceptional, then this subscheme of $X$ is projective over $R / \mathfrak{m}^{n}$ for some $n>0$. In particular, the exceptional prime divisors $E^{1}, E^{2}, \ldots, E^{s}$ are isomorphic to the projective line $\mathbb{P}_{R / \mathfrak{m}}^{1}$; and any two of them intersect transversally. (This can easily be shown by induction on the number of blowups making up $f$.)

For any invertible $\mathcal{O}_{X}$-module $\mathcal{L}$ the intersection number $\mathcal{L} \cdot E$ of $\mathcal{L}$ with an effective $f$-divisor $E$ is the degree of the invertible $\mathcal{O}_{E}$-module $\mathcal{L}_{E}:=\mathcal{L} \otimes \mathcal{O}_{E}$ :

$$
\mathcal{L} \cdot E:=\chi_{E} \mathcal{L}_{E}-\chi_{E} \mathcal{O}_{E}
$$

where with $\lambda$ the length function on $R$-modules,

$$
\chi_{E} \mathcal{M}:=\lambda \mathrm{H}^{0}(E, \mathcal{M})-\lambda \mathrm{H}^{1}(E, \mathcal{M})
$$

is the Euler characteristic of a coherent $\mathcal{O}_{E}$-module $\mathcal{M}$.

For any $X$-divisor $F$ set $F \cdot E:=\mathcal{O}_{X}(F) \cdot E$. This intersection number extends uniquely to a $\mathbb{Z}$-valued symmetric bilinear form on $\operatorname{Div}_{\mathrm{e}}(X)$ (see e.g., [L1, §13]). If $F^{\prime}=(t)+F$ for some $t \in L$ then $\mathcal{O}_{X}\left(F^{\prime}\right) \cong \mathcal{O}_{X}(F)$, and hence $F^{\prime} \cdot E=F \cdot E$.

An $X$-divisor $F$ is said to be numerically effective, nef for short, if $F \cdot E^{i} \geq 0$ for all $i=1,2, \ldots, s \quad\left(\Rightarrow F \cdot E \geq 0\right.$ for all effective $\left.E \in \operatorname{Div}_{\mathrm{e}}(X)\right)$. $F$ is said to be antinef if $-F$ is nef.

The following basic result is contained in [L1, p. 220, Thm. (12.1)].

Theorem 1.1. An $X$-divisor $D$ is nef if and only if $\mathcal{O}_{X}(D)$ is generated by its global sections. 
Corollary 1.1.1. If $D$ is antinef and $D_{\text {aff }} \geq 0$ then $D$ is effective.

That's because, as above, $\Gamma\left(X, \mathcal{O}_{X}(-D)\right) \subset R$, and so by the Theorem,

$$
\mathcal{O}_{X}(-D)=\Gamma\left(X, \mathcal{O}_{X}(-D)\right) \mathcal{O}_{X} \subset \mathcal{O}_{X}
$$

(A simpler proof, not using Theorem 1.1, can be found in [L1, p. 238].)

If $I$ is a complete ideal and $I \mathcal{O}_{X}$ is invertible then $I=\Gamma\left(X, I \mathcal{O}_{X}\right)$, whence:

Corollary 1.1.2. (Cf. $[\mathrm{L} 1, \S 18]$.) Sending $E$ to $\Gamma\left(X, \mathcal{O}_{X}(-E)\right)$ defines an isomorphism from the (additive) monoid of antinef $X$-divisors to the (multiplicative) monoid of those complete ideals $I$ such that $I \mathcal{O}_{X}$ is invertible. ${ }^{2}$ Under this isomorphism antinef $f$-divisors correspond to finite-colength complete ideals.

It is simple to show, by induction on the number of blowups making up $f$, that the intersection matrix $\left(E^{i} \cdot E^{j}\right)$ has determinant \pm 1 . Hence for each $i$ there is a unique $f$-divisor $G_{i}$ such that $G_{i} \cdot E^{j}=0$ unless $j=i$, in which case $G_{i} \cdot E^{j}=-1$; and for any $f$-divisor $E$ it holds that $-E=\sum_{i=1}^{s}\left(E \cdot E^{i}\right) G_{i}$. Thus the monoid of antinef $f$-divisors is freely generated by these $G_{i}$. In other words, "unique factorization" holds in this monoid - and therefore in the monoid of finite-colength complete ideals to which, by Corollary 1.1.2, it is isomorphic.

An integral ideal $P \neq R$ is simple if whenever $P=I J$ ( $I, J$ integral ideals) then either $I$ or $J$ is the unit ideal. For example, if $G_{i}$ is as above then the $\mathfrak{m}$-primary ideal $P_{i}:=\Gamma\left(X, \mathcal{O}_{X}\left(-G_{i}\right)\right)$ is simple, since if $P_{i}$ is the product of $I$ and $J$ then it is also the product of the integral closures of $I$ and $J$.

Corollary 1.1.3. (Zariski, [ZS, p. 386, Thm.3].) Every complete integral ideal is, in a unique way, the product of simple complete ideals.

(As $R$ is a unique factorization domain, one reduces at once to the finitecolength case. Also, it helps to note that for any ideals $I, J$, if $I J \mathcal{O}_{X}$ is invertible then $I \mathcal{O}_{X}$ and $J \mathcal{O}_{X}$ are both invertible.)

Corollary 1.1.4. If $I$ is a complete ideal with $I \mathcal{O}_{X}$ invertible, $I \mathcal{O}_{X}=\mathcal{O}_{X}(-E)$, and $P_{i}:=\Gamma\left(X, \mathcal{O}_{X}\left(-G_{i}\right)\right)$ is the simple $\mathfrak{m}$-primary complete ideal corresponding to the above $G_{i}$, then $I I^{-1}=\prod_{i=1}^{s} P_{i}^{-E} E^{i}$. Thus $P_{i}$ divides $I I^{-1} \Leftrightarrow E \cdot E^{i} \neq 0$.

Moreover, the valuations $v_{E^{i}}$ associated to those $E^{i}$ such that $E \cdot E^{i} \neq 0$ are precisely the Rees valuations of $I I^{-1}$ (i.e., those valuations whose valuation ring is the local ring of the generic point of some reduced irreducible component of the closed fiber of the normalized blowup of $I$ ). (See [L4, p. 300, Prop. (4.4)].)

The following Lemma ${ }^{3}$ will be needed.

\footnotetext{
${ }^{2}$ It is a theorem of Zariski that a product of two complete ideals is still complete [ZS, p. 385, Thm. 2'], [L1, p. 209, Thm. (7.1)].

${ }^{3}$ related to Enriques's "principle of discharge" [Z, p. 28],
} 
Lemma 1.2. Let $E$ be an $X$-divisor and $I$ the complete ideal $\Gamma\left(X, \mathcal{O}_{X}(-E)\right)$. Then $I \mathcal{O}_{X}$ is invertible. Equivalently (see (1.1.2)), there is an antinef $E^{-} \geq E$, such that $I=\Gamma\left(X, \mathcal{O}_{X}\left(-E^{-}\right)\right)$; this $E^{-}$must be the least antinef divisor $\geq E$.

Proof. Since $R$ is a unique factorization domain, there exists a $t \in L$ such that $(t)_{\text {aff }}=E_{\text {aff }}$, and one can replace $(E, I)$ by $\left(E-(t), t^{-1} I\right)$; so one may assume that $E$ is exceptional, say $E=\sum a_{i} E^{i}$, and that $I$ has finite colength. One may also assume $X \neq \operatorname{Spec}(R)$. Then there exists an antinef $F \geq E$ : pick $n$ such that $\mathfrak{m}^{n} \subset I$, and define $F$ by $\mathfrak{m}^{n} \mathcal{O}_{X}=\mathcal{O}_{X}(-F)$. Among all antinef $F=\sum b_{i} E^{i} \geq E$ choose one - call it $F_{E}$ - for which $\sum_{i}\left(b_{i}-a_{i}\right)$ has minimal value, denoted $\sigma_{E}$.

Procede by induction on $\sigma_{E}$. Suppose $\sigma_{E}>0$ (otherwise there is nothing to prove), and that the Lemma holds for all $E^{\prime}$ with $\sigma_{E^{\prime}}<\sigma_{E}$. With $F:=F_{E}$ as above, there is an $i$ such that $E \cdot E^{i}>0 \geq F \cdot E^{i}$, and since $E^{j} \cdot E^{i} \geq 0$ when $j \neq i$ (clearly) and $E^{i} \cdot E^{i}<0,{ }^{4}$ therefore $b_{i}>a_{i}$, whence $F \geq E+E^{i}$. So $\sigma_{E+E^{i}}<\sigma_{E}$, and therefore $\Gamma\left(X, \mathcal{O}_{X}\left(-E-E^{i}\right)\right) \mathcal{O}_{X}$ is invertible. It suffices then to verify that $\Gamma\left(X, \mathcal{O}_{X}\left(-E-E^{i}\right)\right)=I$, by applying the left-exact functor $\Gamma(X,-)$ to the natural exact sequence

$$
0 \longrightarrow \mathcal{O}_{X}\left(-E-E^{i}\right) \longrightarrow \mathcal{O}_{X}(-E) \longrightarrow \mathcal{O}_{X}(-E) \otimes \mathcal{O}_{E^{i}}
$$

and observing that since $\mathcal{O}_{X}(-E) \otimes \mathcal{O}_{E^{i}}$ has degree $-E \cdot E^{i}<0$, therefore

$$
\Gamma\left(X, \mathcal{O}_{X}(-E) \otimes \mathcal{O}_{E^{i}}\right)=\Gamma\left(E^{i}, \mathcal{O}_{X}(-E) \otimes \mathcal{O}_{E^{i}}\right)=0 .
$$

1.3. (Canonical divisors.) Let $Y \stackrel{g}{\rightarrow} X \stackrel{f}{\rightarrow} \operatorname{Spec}(R)$ be proper birational maps with $Y$ and $X$ regular schemes. By a theorem of Zariski and Abhyankar (see, e.g., [L1, p. 204, Thm. (4.1)]) both $f$ and $g$ are compositions of point blowups.

Let $F^{1}, F^{2}, \ldots, F^{t}$ be the reduced irreducible components of $(f g)^{-1}\{\mathfrak{m}\}$. As before, the intersection matrix $\left(F^{i} \cdot F^{j}\right)$ has determinant \pm 1 , and so there is a unique $f g$-divisor $K_{g}$ such that

$$
K_{g} \cdot F^{i}= \begin{cases}-F^{i} \cdot F^{i}-2 & \text { if } g\left(F^{i}\right) \text { is a point } \\ 0 & \text { otherwise. }\end{cases}
$$

This $K_{g}$ is called the canonical divisor of $g .^{5}$

For an $X$-divisor $D, g^{*} D$ denotes the $Y$-divisor whose coefficient at any prime divisor $F$ on $Y$ is $v_{F}\left(\mathcal{O}_{X}(-D)_{x}\right)$, where $x \in X$ is the $g$-image of the generic point of $F$. There is a natural isomorphism $g^{*} \mathcal{O}_{X}(D) \stackrel{\sim}{\longrightarrow} \mathcal{O}_{Y}\left(g^{*} D\right)$.

1.3.2. The following easily-checked properties characterize $K_{g}$ for all $g$ :

- If $g$ is the blowup of a closed point $x \in X$ then $K_{g}=g^{-1}\{x\}$.

- If $Z \stackrel{h}{\rightarrow} Y \stackrel{g}{\rightarrow} X \stackrel{f}{\rightarrow} \operatorname{Spec}(R)$ are proper birational maps with $Z, Y$ and $X$ regular schemes, then

$$
K_{g h}=h^{*} K_{g}+K_{h} .
$$

\footnotetext{
${ }^{4}$ It is well-known, going back to $\mathrm{Du}$ Val, that the intersection matrix $\left(E^{i} \cdot E^{j}\right)$ is negative definite, see e.g., [L1, p. 224, Lemma (14.1)].

${ }^{5}$ One has $\mathcal{O}\left(K_{g}\right)=H^{0}\left(g^{!} \mathcal{O}_{X}\right)$ with $g^{!}$as in Grothendieck duality theory [LS, p. 206, (2.3)].
} 
1.4. (Multiplier ideals.) For $D=\sum_{E} d_{E} E \in \operatorname{Div}(X) \otimes_{\mathbb{Z}} \mathbb{R}$ set

$$
[D]:=\sum_{E}\left[d_{E}\right] E \in \operatorname{Div}(X)
$$

where $\left[d_{E}\right]$ is the greatest integer $\leq d_{E}$.

Definition 1.4.1. Let $I$ be a complete ideal, $h: Y \rightarrow \operatorname{Spec}(R)$ a log resolution of $I$, say $I \mathcal{O}_{Y}=\mathcal{O}_{Y}(-G)$, and let $c$ be a positive real number. The multiplier ideal $\mathcal{J}\left(I^{c}\right)$ is defined to be

$$
\mathcal{J}\left(I^{c}\right):=\Gamma\left(Y, \mathcal{O}\left(K_{h}-[c G]\right)\right)
$$

Thus, by Lemma 1.2,

$$
\mathcal{J}\left(I^{c}\right) \mathcal{O}_{Y}=\mathcal{O}_{Y}(-F)
$$

where $F:=\left([c G]-K_{h}\right)^{-}$is the least antinef $h$-divisor $\geq[c G]-K_{h}$.

For the blowup $h_{1}: Y_{1} \rightarrow Y$ of a closed point $y \in Y$ one finds via (1.3.2) that the $\log$ resolution $h$ can be replaced by the $\log$ resolution $h \circ h_{1}$ without affecting $\mathcal{J}\left(I^{c}\right)$. (When $c$ is not an integer, the log-resolution hypothesis that $G$ be a "normal crossing divisor" is important.) As any two log resolutions are dominated by a third, obtained from each of the two by a sequence of point blowups, ${ }^{6}$ it follows that $\mathcal{J}\left(I^{c}\right)$ does not depend on the choice of the log resolution $h$.

Corollary 1.4.2. A complete ideal $J$ satisfies $J=\mathcal{J}\left(I^{c}\right)$ for some $c, I$ iff for some $\log$ resolution $h: Y \rightarrow \operatorname{Spec}(R)$ of $J$, say $J \mathcal{O}_{Y}=\mathcal{O}_{Y}(-F)$, there is an antinef h-divisor $G$ and a real $c>0$ such that

$$
F=\left([c G]-K_{h}\right)^{-}
$$

\section{Proof of Main Result}

Let $J$ be a complete ideal. To show that $J=\mathcal{J}\left(I^{c}\right)$ for some $c, I$, we will describe a $\log$ resolution $h: Y \rightarrow \operatorname{Spec}(R)$ of $J$, and a $G$ and $c$ as in Corollary 1.4.2, such that if $J \mathcal{O}_{Y}=\mathcal{O}_{Y}(-F)$ then (1.4.2.1) holds. (The number of suitable $(h, G, c)$ will be enormous.)

Factor $J J^{-1}$ as $J J^{-1}=\prod_{\ell=1}^{u} P_{\ell}^{e_{\ell}}\left(P_{\ell}\right.$ simple complete, $\left.e_{\ell}>0\right)$-see Corollary 1.1.3 and the paragraph preceding it. Let $f: X \rightarrow \operatorname{Spec}(R)$ be any $\log$ resolution of $J$, say $J \mathcal{O}_{X}=\mathcal{O}_{X}\left(-F^{0}\right)$. As in Corollary 1.1.4, one has for each $\ell$,

$$
e_{\ell}=-F^{0} \cdot E^{\ell}
$$

\footnotetext{
${ }^{6} \mathrm{By}$ the above-mentioned theorem of Zariski and Abhyankar, it suffices to principalize some ideal sheaf on one of the log resolutions by a sequence of point blowups ("elimination of indeterminacies"), which can be done e.g., via the Hoskin-Deligne formula, as before.
} 
We will first construct, for each $u$-tuple $N:=\left(n_{1}, n_{2}, \ldots, n_{u}\right)$ of non-negative integers, a proper birational map $g_{N}: Y_{N} \rightarrow X$, to be realized as a composition of closed point blowups, so that $h_{N}:=f \circ g_{N}$ will be a $\log$ resolution of $J$.

For ease of expression we say "blow up a closed point $x \in X$ generically, $n$ times" to mean "blow up $x_{0}:=x$ to get $g_{1}: X_{1} \rightarrow X$, then blow up a closed point $x_{1}$ on $g_{1}^{-1} x_{0}$ but not on any other irreducible component of $g_{1}^{*} F^{0}$ to get $g_{2}: X_{2} \rightarrow X_{1}$, then blow up a closed point $x_{2}$ on $g_{2}^{-1} x_{1}$ but not on $\ldots$ then blow up a closed point $x_{n-1}$ on $g_{n-1}^{-1} x_{n-2}$ but not on any other irreducible component of $\left(g_{1} \circ g_{2} \circ \cdots \circ g_{n-1}\right)^{*} F^{0}$ to get $g_{n}: X_{n} \rightarrow X_{n-1}$."

Then with $g:=g_{1} \circ g_{2} \circ \cdots \circ g_{n}$ it holds that:

2.1. $g^{-1} x$ is a chain of $n$ integral curves $D_{1}, D_{2}, \ldots, D_{n}$ such that for $0<i<n$, $D_{i} \cdot D_{i+1}=1$ and $D_{i} \cdot D_{i}=-2$, while $D_{n} \cdot D_{n}=-1$; and if $|j-i|>1$ then $D_{i} \cdot D_{j}=0$.

(For the proof one can use, e.g., [L1, p. 229, middle, and p. 227, $\alpha$ ) and $\beta$ )]. Here, and subsequently, the reader may find it useful to do some rough sketches.)

As in Corollary 1.1.4, there corresponds to each $D_{i}$ an $\mathfrak{m}$-primary simple complete ideal $Q_{i}$; and, we claim, these $Q_{i}$ form a strictly decreasing sequence $Q_{1}>Q_{2}>\cdots>Q_{n}$, with $Q_{1}$ strictly contained in each of the simple ideals corresponding to the (one or two) prime $f$-divisors $E^{j}$ passing through $x$.

Indeed, let $G_{j}$ be the $f$-divisor such that $G_{j} \cdot E^{j}=-1$ and $G_{j} \cdot E=0$ for every other prime $f$-divisor $E$, and let $\widetilde{Q}_{j}:=\Gamma\left(X, \mathcal{O}_{X}\left(-G_{j}\right)\right)$ be the corresponding simple complete ideal. It follows from, e.g., $[\mathrm{L} 1$, p. $227, \alpha)$ and $\beta)]$ that $g_{1}^{*} G_{j}$ is antinef; and the corresponding simple complete ideal is

$$
\Gamma\left(X_{1}, \mathcal{O}_{X_{1}}\left(-g_{1}^{*} G_{j}\right)\right)=\Gamma\left(X, \mathcal{O}_{X}\left(-G_{j}\right)\right)=\widetilde{Q}_{j}
$$

Further, with $E^{\prime}:=g_{1}^{-1} x$, let $G^{\prime}$ be the $f g_{1}$-divisor such that $G^{\prime} \cdot E^{\prime}=-1$ and $G^{\prime} \cdot E^{\prime \prime}=0$ for every other prime $f g_{1}$-divisor $E^{\prime \prime}$. Since $g_{1}^{*} G_{j}+E^{\prime}$ has intersection number -1 with $E^{\prime}$ and $\geq 0$ with each $E^{\prime \prime}$, therefore $G^{\prime}-g_{1}^{*} G_{j}-E^{\prime}$ is antinef, hence effective (Corollary 1.1.1); and consequently $G^{\prime}>g_{1}^{*} G_{j}$. Thus the simple complete ideal $\Gamma\left(X_{1}, \mathcal{O}_{X_{1}}\left(-G^{\prime}\right)\right)$ is strictly contained in $\widetilde{Q}_{j}$.

Continuing in this way we establish the claim.

Now for each $\ell=1,2, \ldots, u$, pick $e_{\ell}$ distinct closed points $x_{1}, \ldots, x_{e_{\ell}}$ which lie on $E^{\ell}$ but on no $E \neq E^{\ell}$ and blow up all of these points generically, $n_{\ell}$ times. Then $Y_{N}$ is the resulting surface, and $g_{N}$ is the composition of all the blowups. It is easily seen that $\left(Y_{N}, g_{N}\right)$ does not depend (up to isomorphism) on the order in which the chosen points are blown up - though that won't be important. ${ }^{7}$

\footnotetext{
${ }^{7}$ The initial $\sum_{\ell} e_{\ell}$ points might be taken to be the intersection of the closed fiber on $X$ with a sufficiently generic curve $C$ in the linear system $\left|-F^{0}\right|$ (i.e., a divisor-having no component in the closed fiber - of the form $(j)-F^{0}$ with $j$ a sufficiently generic element of $J$ ). Then at each stage the point to be blown up could be taken to be a specialization of some nonclosed point of $C$.
} 
To simplify notation, fix $N$ and set $(Y, g):=\left(Y_{N}, g_{N}\right)$ and $F:=g^{*} F^{0}$, so that $J \mathcal{O}_{Y}=\mathcal{O}_{Y}(-F)$. Also, set $h:=f g: Y \rightarrow \operatorname{Spec}(R)$.

For an $X$-divisor $D$, we denote by $D^{\#}$ the proper transform of $D$ on $Y$, obtained from $g^{*} D=: \sum_{E} a_{E} E$ (where $E$ runs through all prime divisors on $Y$ ) by replacing $a_{E}$ by 0 whenever $E$ is $g$-exceptional, i.e., $g(E)$ is a closed point.

For each $\ell=1,2, \ldots, u$ and $x_{j_{\ell}} \in E^{\ell}\left(j_{\ell}=1,2, \ldots, e_{\ell}\right)$ let

$$
\left\{E_{j_{\ell} k_{\ell}}^{\ell} \mid k_{\ell}=1,2, \ldots, n_{\ell}\right\}
$$

be the family of prime $Y$-divisors whose $g$-image is $x_{j_{\ell}}$, the ordering of these curves by the index $k_{\ell}$ conforming to the ordering of the $D$ 's in (2.1). These curves are all isomorphic to the projective line $\mathbb{P}_{R / \mathfrak{m}}^{1}$.

If $a_{\ell}$ is the $E^{\ell}$-coefficient of the divisor $F^{0}$, and $b_{\ell}$ of the divisor $K_{f}$, then one finds (using (1.3.2)) that

$$
\begin{aligned}
F=g^{*} F^{0} & =F^{0 \#}+\sum_{\ell} \sum_{j_{\ell}, k_{\ell}} a_{\ell} E_{j_{\ell} k_{\ell}}^{\ell}, \\
g^{*} K_{f} & =K_{f}^{\#}+\sum_{\ell} \sum_{j_{\ell}, k_{\ell}} b_{\ell} E_{j_{\ell} k_{\ell}}^{\ell} \\
K_{g} & =\sum_{\ell} \sum_{j_{\ell}, k_{\ell}} k_{\ell} E_{j_{\ell} k_{\ell}}^{\ell}
\end{aligned}
$$

Set $G:=F+K_{g}$. Noting that $\left.F \cdot E_{j_{\ell} k_{\ell}}^{\ell}=g^{*} F^{0} \cdot E_{j_{\ell} k_{\ell}}^{\ell}=0[\mathrm{~L} 1, \mathrm{p} .227, \beta)\right]$, and using (2.1) together with the preceding expansion of $K_{g}$ (or together with (1.3.1) and (1.3.2)), one finds that for every $g$-exceptional prime divisor $E, G \cdot E=0$ unless $E$ is one of the curves $E_{j_{\ell} n_{\ell}}^{\ell}$ at the end of the chains emanating from the $\sum_{\ell} e_{\ell}$ originally chosen points (i.e., $g(E)$ is a point and $E \cdot E=-1$ ), in which case $G \cdot E=-1$. Moreover, for any $f$-exceptional prime divisor $D, g^{*} F^{0} \cdot D^{\#}=F^{0} \cdot D$ $[\mathrm{L} 1, \mathrm{p} .227, \alpha)]$, and $E_{j_{\ell} k_{\ell}}^{\ell} \cdot D^{\#}=0$ if $k_{\ell}>1$ (since then $E_{j_{\ell} k_{\ell}}^{\ell} \cap D^{\#}$ is empty), so using (2.0) one finds that $G \cdot D^{\#}=0$. Thus $G$ is an antinef $Y$-divisor.

By Corollary 1.1.4, if $I$ is the corresponding complete ideal $\Gamma\left(Y, \mathcal{O}_{Y}(-G)\right)$ then $I I^{-1}$ is the product of the simple complete ideals corresponding to the $\sum_{\ell} e_{\ell}$ curves $E_{j_{\ell} n_{\ell}}^{\ell}$ having self-intersection -1 .

Here is a key technical point:

Lemma 2.2. For all sufficiently small $\epsilon>0$ there exists $N$ such that

$$
(1+\epsilon) G-K_{h}=F+A \quad([A] \leq 0)
$$

where the coefficient of $[A]$ at each $E_{j_{\ell} n_{\ell}}$ and at each affine prime $Y$-divisor is 0.

Proof. Using (1.3.2), one transforms (2.2.1) into the equality

$$
\epsilon\left(F+K_{g}\right)-g^{*} K_{f}=A .
$$


More explicitly (see above)

$$
\epsilon\left(F^{0 \#}+\sum_{\ell} \sum_{j_{\ell}, k_{\ell}}\left(a_{\ell}+k_{\ell}\right) E_{j_{\ell} k_{\ell}}^{\ell}\right)-\left(K_{f}^{\#}+\sum_{\ell} \sum_{j_{\ell}, k_{\ell}} b_{\ell} E_{j_{\ell} k_{\ell}}^{\ell}\right)=A .
$$

So to get (2.2.1) we can choose any $\epsilon>0$ such that the coefficients of the $X$-divisor $\epsilon F^{0}-K_{f}$ are all $<1$, and then look for $n_{\ell}$ such that $\epsilon\left(a_{\ell}+k_{\ell}\right)-b_{\ell}<1$ for all $\ell$ and for all $k_{\ell} \leq n_{\ell}$, while $\epsilon\left(a_{\ell}+n_{\ell}\right)-b_{\ell} \geq 0$. These conditions mean precisely that $n_{\ell}$ satisfies the inequalities

$$
1 / \epsilon+b_{\ell} / \epsilon-a_{\ell}>n_{\ell} \geq b_{\ell} / \epsilon-a_{\ell} \quad(\ell=1,2, \ldots, u) .
$$

Clearly, such integers $n_{\ell}$ can be found if $\epsilon<1$.

For $c=1+\epsilon$ and $N$ satisfying Lemma 2.2, and with $h: Y \rightarrow \operatorname{Spec}(R)$ and $F, G$, as before, we have

$$
F^{\prime}:=\left([c G]-K_{h}\right)^{-} \leq F
$$

so that

$$
J^{\prime}:=\Gamma\left(Y, \mathcal{O}\left(-F^{\prime}\right)\right) \supset \Gamma(Y, \mathcal{O}(-F))=J .
$$

Let us verify that $J^{\prime}=J\left(=\left(J^{-1}\right)^{-1} J J^{-1}\right)$, thereby proving the main result.

Since $G=F+K_{g}$ and $F$ have the same affine part, the affine part of $[A]$ must be 0 , and hence $F^{\prime}$ and $F$ have the same affine part. This means that $\left(J^{\prime-1}\right)^{-1}=\left(J^{-1}\right)^{-1}$. So $J^{\prime} J^{\prime-1} \supset J J^{-1}$, and we need only show that these two $\mathfrak{m}$-primary ideals are equal.

Recall that the valuations $v_{\ell}:=v_{E^{\ell}}$ are just the Rees valuations of $J J^{-1}$. (See the remark following Corollary 1.1.4). So

$$
J J^{-1}=\left\{\rho \in R \mid v_{\ell}(\rho) \geq v_{\ell}\left(J J^{-1}\right) \text { for all } \ell=1,2, \ldots, u\right\} .
$$

Thus we need only show that for each $\ell$, the $E^{\ell \#}$-coefficient $a_{\ell}^{\prime}$ of $F^{\prime}$ is the same as that of $F$ (namely $a_{\ell}$ ). Let us say that $\ell$ is "good" if $a_{\ell}^{\prime}=a_{\ell}$ and "bad" if $a_{\ell}^{\prime}<a_{\ell}$.

If $\ell$ is good then since $F^{\prime} \leq F$ therefore

$$
F^{\prime} \cdot E^{\ell \#} \leq F \cdot E^{\ell \#}=F^{0} \cdot E^{\ell} \stackrel{(2.0)}{=}-e_{\ell} .
$$

Corollary 1.1.4 shows then that $J^{\prime} J^{\prime-1}$ is divisible by $P_{\ell}^{e_{\ell}}$.

Suppose $\ell$ is bad. For $j \in\left[1, e_{\ell}\right]$ and with $a_{j k}^{\prime}$ the $E_{j k}^{\ell}$-coefficient of $F^{\prime}$ it is easily seen that $a_{\ell}^{\prime}=: a_{j 0}^{\prime} \leq a_{j 1}^{\prime} \leq a_{j 2}^{\prime} \leq \cdots \leq a_{j n_{\ell}}^{\prime}=a_{\ell}{ }^{8}$ So there is a $k \in\left[1, n_{\ell}\right]$ such that $a_{j, k-1}^{\prime}<a_{j k}^{\prime}=a_{j, k+1}^{\prime}=\cdots=a_{\ell}$. Then

$$
F^{\prime} \cdot E_{j k}^{\ell}= \begin{cases}a_{j, k-1}^{\prime}-2 a_{j k}^{\prime}+a_{j, k+1}^{\prime}<0 & \text { if } k<n_{\ell}, \\ a_{j, n_{\ell}-1}^{\prime}-a_{j n_{\ell}}^{\prime}<0 & \text { if } k=n_{\ell} .\end{cases}
$$

\footnotetext{
${ }^{8}$ With $v:=v_{E_{j, k+1}^{\ell}}$ and $S \supset R$ the regular local ring blown up to give $E_{j, k+1}^{\ell}$, one finds: $a_{j, k+1}^{\prime}=v\left(J^{\prime} S\right)=v\left(\left(\left(J^{\prime} S\right)^{-1}\right)^{-1}\right)+v\left(J^{\prime} S\left(J^{\prime} S\right)^{-1}\right)=a_{j k}^{\prime}+v\left(J^{\prime} S\left(J^{\prime} S\right)^{-1}\right)$.
} 
From Corollary 1.1.4 and the remarks after 2.1, one deduces that $J^{\prime} J^{\prime-1}$ is divisible by a simple complete ideal $P_{\ell j}^{\prime}<P_{\ell}$. This being so for all $j$, and the $P_{\ell j}^{\prime}$ being distinct (Corollary 1.1.2), it follows from Corollary 1.1.3 that $J^{\prime} J^{\prime-1}$ is divisible by $P_{\ell 1}^{\prime} P_{\ell 2}^{\prime} \cdots P_{\ell e_{\ell}}^{\prime}<P_{\ell}^{e_{\ell}}$. Thus (by Corollary 1.1.3 again) the existence of a bad $\ell$ leads to a factorization of $J^{\prime} J^{\prime-1}$ which contradicts $J^{\prime} J^{\prime-1} \supset J J^{-1}$.

So every $\ell$ is good, and $J^{\prime} J^{\prime-1}=J J^{-1}$.

\section{Remarks.}

1. By the choice of $\epsilon$, the $E^{\ell}$-coefficient of $\epsilon F^{0}-K_{f}$ is $<1$, i.e., $\epsilon a_{\ell}-b_{\ell}<1$, i.e., $b_{\ell} / \epsilon-a_{\ell}>-1 / \epsilon$. It could happen that $b_{\ell} / \epsilon-a_{\ell}<0$ for all $\ell$. In this case one can take $N=(0,0, \ldots, 0)$, and then $J=\mathcal{J}((1+\epsilon) J)$.

2. The proof shows that if $J$ is a simple complete $\mathfrak{m}$-primary ideal then there is a simple complete $\mathfrak{m}$-primary ideal $P \subset J$ and a $c>0$ such that $J=\mathcal{J}(c P)$.

3. By way of illustration of our method, let $J$ be a simple $\mathfrak{m}$-primary ideal of order 16, whose successive transforms have orders $(8,8,4,4,2,2,1$, $1,0,0 \ldots)$. (For the existence of such a $J$ see e.g., [L4, p. 298, Cor. (3.1)].) Here $\ell=1$, and one calculates that $a_{1}=426, b_{1}=46$. Then any $\epsilon \in(0,5 / 48)$ will do. Since $46 /(5 / 48)-426>15$, the least possible value of $N$ is 16 , which is attained, e.g., when $\epsilon=23 / 221$. One has then that $J=\mathcal{J}\left(P^{244 / 221}\right)$, where $P \subset J$ is a simple complete ideal of order 16 with successive transforms of orders $(8,8,4,4,2,2,1,1, \ldots, 1,0,0 \ldots)(18$ ones).

A simpler permissible choice of $\epsilon$ would be $1 / 12$. But one would need $N=126$ blowups to unearth a simple $P \subset J$ such that $J=\mathcal{J}\left(P^{13 / 12}\right)$.

There might be other methods of finding a representation $J=\mathcal{J}\left(I^{c}\right)$ with $I$ "closer" to $J$ than here. But $I=J$ can never occur, because the inequality $b_{1}<\epsilon a_{1}$ of example 1 could then be deduced. (Exercise.) On the other hand, this inequality does hold for any of the successive transforms of the present $J$.

4. Since the $c=1+\epsilon$ we have considered can be arbitrarily close to 1 , one may ask if it is possible for $c$ actually to be 1 . (This would be the case studied in [L5], where $\mathcal{J}(I)$ is called the adjoint ideal of $I$.)

For simple complete $\mathfrak{m}$-primary $J$, the answer is given by:

Proposition 2.3. A simple complete $\mathfrak{m}$-primary ideal $J$ is of the form $\mathcal{J}(I)$ for some $I \Longleftrightarrow J \not \subset \mathfrak{m}^{2} \Longleftrightarrow J=\left(a, b^{n}\right) R$ for some integer $n>0$ and $a, b \in R$ such that $(a, b) R=\mathfrak{m}$.

Proof. The last $\Longleftrightarrow$ holds because $J \not \subset \mathfrak{m}^{2}$ means that $J$ contains an element $a$ such that $R / a R$ is a discrete valuation ring. Moreover, if $(a, b) R=\mathfrak{m}$ and $z \in R$ is integral over $J=\left(a, b^{n}\right) R$ then the canonical image of $z$ in $R / a R$ is integral over-and hence is a multiple of - that of $b^{n}$, whence $z \in J$, and thus $J$ is complete (and clearly simple). It is an easy exercise to show that for such a $J$, $J=\mathcal{J}\left(J^{2}\right)$. (One could use [L5, p. 749, Prop. (3.1.2)].) 
For a simple complete m-primary $J$, let $f: X \rightarrow \operatorname{Spec}(R)$ be a log resolution obtained by successively blowing up base points of $J$-closed points at which $J$ does not generate an invertible ideal - for as long as such points are available. (As noted before, the Hoskin-Deligne formula guarantees that this process terminates.) From [L1, p. 199, Prop. 1.2 and p. 203, Prop. 3.1] it follows that this $f$ is the minimal desingularization of the blowup of $J$ : for any $\log$ resolution $h$ of $J$ there exists a map $g: Y \rightarrow X$ composed of point blowups such that $h=f g$.

We have seen before that there is a unique exceptional prime $X$-divisor $E$ such that $J \mathcal{O}_{X} \cdot E \neq 0$. This $E$ satisfies $E \cdot E=-1$ : for, there is at least one exceptional prime $X$-divisor $E^{\prime}$ such that $E^{\prime} \cdot E^{\prime}=-1$, namely the closed fibre $f^{\prime-1}\left\{x^{\prime}\right\}$ for the blowup $f^{\prime}: X \rightarrow X^{\prime}$ of $x^{\prime} \in X^{\prime}$ coming last in the sequence of blowups composing to $f$ (see [L1, middle of p. 229]); and $J^{\prime}:=J \mathcal{O}_{X^{\prime}, x^{\prime}}$ is not invertible, from which one sees, with $\mathfrak{m}^{\prime}$ the maximal ideal of $R^{\prime}:=\mathcal{O}_{X^{\prime}, x^{\prime}}$, that $J^{\prime}=d \mathfrak{m}^{\prime s}$ for some $d \in R^{\prime}$ and $s>0$, whence $J \mathcal{O}_{X} \cdot E^{\prime}=s$, so that $E^{\prime}=E$.

We claim that if $F \cdot F=-2$ for all exceptional prime divisors $F \neq E$ then $J \not \subset \mathfrak{m}^{2}$. (The converse is part of the exercise at the end of the first paragraph.) Indeed, this condition on the $F$ 's means that among the base points of $J$ no two are "proximate" to the same one, and the conclusion follows from [L4, p. 301, (3)].

Assume now that $J=\mathcal{J}(I)$. From $J$ being $\mathfrak{m}$-primary it follows easily that so is $I$. ( $I$ and $\mathcal{J}(I)$ have the same gcd.) Let $h: Y \rightarrow \operatorname{Spec}(R)$ be a log resolution of $I$, obtained as above by blowing up base points of $I$, and say $I \mathcal{O}_{Y}=\mathcal{O}_{Y}(-G)$. Let $F$ be an exceptional prime $Y$-divisor. As above, we find that $F \cdot F=-1 \Rightarrow G \cdot F<0$. (We may assume that $F$ is the closed fiber for the last blowup in some sequence of blowups composing to $g$, because if $g$ is the blowup of a point lying on an exceptional prime divisor $F_{1}$ then $0>F_{1} \cdot F_{1}=g^{*} F_{1} \cdot F_{1}^{\#}=F_{1}^{\#} \cdot F_{1}^{\#}+1$.) Since $K_{h} \cdot F=-F \cdot F-2$ and $G$ is antinef, we see that $G-K_{h}$ is antinef, and hence $\mathcal{O}_{Y}\left(K_{h}-G\right)=J O_{Y}$.

So $J \mathcal{O}_{Y}$ is invertible, i.e., $h$ is a $\log$ resolution of $J$, and as above there exists a $g: Y \rightarrow X$ composed of point blowups such that $h=f g$. Let $F^{1 \#}, F^{2 \#}, \ldots, F^{n \#}$ be the proper transforms on $Y$ of the prime $X$-divisors $F^{1}, F^{2}, \ldots, F^{n}$ other than the above $E$. Then $F^{i \#} \cdot F^{i \#} \leq F^{i} \cdot F^{i} \leq-2$ and so $K_{h} \cdot F^{i \#} \geq 0$. But by $[\mathrm{L} 1$, p. $\left.227, \beta)\right]$,

$$
K_{h} \cdot F^{i \#} \leq\left(K_{h}-G\right) \cdot F^{i \#}=J O_{Y} \cdot F^{i \#}=J O_{X} \cdot F^{i}=0,
$$

and thus $K_{h} \cdot F^{i \#}=0$, i.e., $F^{i \#} \cdot F^{i \#}=-2$, whence, finally, $F^{i} \cdot F^{i}=-2$. The above claim shows then that $J \not \subset \mathfrak{m}^{2}$.

\section{References}

[FJ] C. Favre, M. Jonsson, A valuative criterion of integrability, preprint.

[La] R. Lazarsfeld, Positivity in algebraic geometry, draft available at: http://www.math.lsa.umich.edu/ ${ }^{\sim}$ laz/ .

[L1] J. Lipman, Rational singularities, with applications to algebraic surfaces and unique factorization, Inst. Hautes Études Sci. Publ. Math. 36 (1969), 195-279.

[L2] _ On complete ideals in regular local rings, Algebraic geometry and commutative algebra, Vol. I, 203-231, Kinokuniya, Tokyo, 1988. 
[L3] _ Adjoints and polars of simple complete ideals in two-dimensional regular local rings, Bull. Soc. Math. Belg. Sér. A 45 (1993), 223-244.

[L4] _ Proximity inequalities for complete ideals in two-dimensional regular local rings, Contemp. Math. 159 (1994), 293-306.

[L5] _ Adjoints of ideals in regular local rings, Math. Res. Lett. 1 (1994), 739-755.

[LS] J. Lipman, A. Sathaye, Jacobian ideals and a theorem of Briançon-Skoda, Michigan Math. J. 28 (1981), 199-222.

[Z] O. Zariski, Algebraic Surfaces, Second supplemented edition. Springer-Verlag, New YorkHeidelberg, 1971.

[ZS] O. Zariski, P. Samuel, Commutative Algebra, Vol. II. D. Van Nostrand Co., Inc., Princeton, NJ -Toronto-London-New York, 1960.

Dept. of Mathematics, Purdue University, W. Lafayette, IN 47907, U.S.A.

E-mail address: lipman@math.purdue.edu

Dept. of Mathematics, Nihon University, Sakura Josui 3-25-40, Setagaya, Tokyo 156-8550, JAPAN.

E-mail address: watanabe@math.chs.nihon-u.ac.jp 